\title{
Anestesia y diabetes en el perioperatorio
}

\author{
Anesthesia and diabetes in the perioperative period
}

\author{
Jaime Pablo Antonio Ortega García, ${ }^{*}$ Norma Elizabeth Carrillo Molina, ${ }^{*}$ Angélica Yanine López Ramírez ${ }^{\ddagger}$
}

Citar como: Ortega GJPA, Carrillo MNE, López RAY. Anestesia y diabetes en el perioperatorio. An Med ABC. $2021 ; 66$ (3): $195-204$. https://dx.doi.org/10.35366/101667

\begin{abstract}
RESUMEN
La diabetes mellitus es una enfermedad metabólica caracterizada por hiperglucemia crónica y trastornos en el metabolismo de carbohidratos, proteínas y grasas. Afecta alrededor de $9.3 \%$ de la población mundial. En México hasta $25 \%$ de los pacientes quirúrgicos pueden ser diabéticos, siendo una de las primeras causas de morbimortalidad, así como el aumento en el número de días de estancia intrahospitalaria. Durante el manejo intraoperatorio el objetivo principal radica en mantener niveles adecuados de glucosa, concentración normal de electrólitos, así como garantizar la función óptima y adecuada del nivel cardiovascular y renal. Es responsabilidad del equipo quirúrgico contar con los recursos necesarios para realizar el diagnóstico y tratar la hiperglucemia perioperatoria, con el fin de disminuir las complicaciones relacionadas. En casos de cirugía electiva se recomiendan niveles de hemoglobina glucosilada $\leq 8.5 \%$. El objetivo de esta revisión es conocer la fisiopatología de la diabetes en el perioperatorio con el fin de tratar, mejorar y disminuir todas las complicaciones a tiempo.
\end{abstract}

Palabras clave: Diabetes, hiperglucemia, perioperatorio, anestesia.

Nivel de evidencia: III

Abreviaturas:

$C A D=$ Cetoacidosis diabética.

$\mathrm{HbA1c}=$ Hemoglobina glucosilada .

$\mathrm{ADA}=$ Asociación Americana de Diabetes.

$\mathrm{UI}=$ Unidades internacionales.

\footnotetext{
* Médico Anestesiólogo e Intensivista, adscrito al Departamento de Anestesiología.

‡ Anestesióloga Oncóloga, adscrita al Departamento de Anestesiología.
}

\begin{abstract}
Diabetes mellitus is a metabolic disease characterized by chronic hyperglycemia and disorders in the metabolism of carbohydrates, proteins and fats. It affects about $9.3 \%$ of the world population. In Mexico, up to $25 \%$ of surgical patients may be diabetic, being one of the leading causes of morbi and mortality, as well as an increase in the number of days of hospital stay. During intraoperative management, the main objective is to maintain adequate glucose levels, normal electrolyte concentration, as well as to guarantee optimal and adequate cardiovascular and renal function. It is the responsibility of the surgical team to have the necessary resources to diagnose and treat perioperative hyperglycemia, in order to reduce related complications. In cases of elective surgery, glycosylated hemoglobin levels $\leq$ $8.5 \%$ are recommended. The goal of this review is to know the pathophysiology of diabetes in the perioperative period in order to treat, improve and reduce all complications in time.
\end{abstract}

Keywords: Diabetes, hyperglycemia, perioperative, anesthesia.

Level of evidence: III

\author{
Correspondencia: \\ Dra. Angélica Yanine López Ramírez \\ E-mail: yanine3010@gmail.com
}

\section{INTRODUCCIÓN}

La diabetes mellitus es una condición crónica de hiperglucemia que afecta alrededor de $9.3 \%$ de la población mundial. La literatura médica demuestra 
una clara asociación entre hiperglucemia perioperatoria y una evolución clínica tórpida. El riesgo de complicaciones postoperatorias y el incremento en la mortalidad se relacionan con el control de la glucemia y la severidad de la hiperglucemia durante la estancia intrahospitalaria. ${ }^{1-3}$

Los niveles de glucosa en sangre elevados impiden la función habitual de los neutrófilos y causan una sobreproducción de especies de oxígeno reactivas, ácidos grasos libres y mediadores inflamatorios. Estos cambios fisiopatológicos contribuyen a un daño celular directo y disfunción vascular e inmune. ${ }^{4} \mathrm{La}$ evidencia científica indica que la corrección de la hiperglucemia en el perioperatorio reduce las complicaciones hospitalarias y disminuye la mortalidad en pacientes de cirugía general y cardiaca; ${ }^{5,6}$ sin embargo, el manejo óptimo de la glucosa en el periodo perioperatorio es ampliamente discutido.

\section{Concepto}

La diabetes es una enfermedad metabólica caracterizada por hiperglucemia crónica y trastornos en el metabolismo de los carbohidratos, proteínas y grasas como resultado de defectos en la secreción de insulina, en su mecanismo de acción o ambas. ${ }^{7}$

\section{Prevalencia}

La diabetes mellitus tipo 2 es la forma más común y su prevalencia global se eleva como resultado de cambios en el estilo de vida y el aumento de la esperanza de vida. ${ }^{8}$

Se estima que alrededor de 463 millones de personas entre 20 y 79 años tienen diabetes, ${ }^{9}$ lo cual corresponde a $9.3 \%$ de la población adulta y representa cuatro veces un incremento en la prevalencia de la diabetes desde $1980 .{ }^{10}$ Pacientes con diabetes tipo 2 frecuentemente tienen comorbilidades como hipertensión arterial, obesidad, enfermedad isquémica y ateroesclerosis. La enfermedad coronaria, en particular, puede afectar a pacientes jóvenes comparada con la población no diabética y puede existir isquemia silenciosa; la valoración del riesgo preoperatorio de los pacientes diabéticos es un reto y pueden ser subestimados, tienen mayor riesgo para eventos cardiovasculares y mortalidad que los pacientes con angina típica. ${ }^{2}$

Las guías actuales recomiendan pruebas de estrés en individuos diabéticos asintomáticos. ${ }^{11}$ Aunque la hiperglucemia perioperatoria es reportada hasta en 20 a $40 \%$ de pacientes para cirugía general, y aproximadamente $80 \%$ en pacientes después de cirugía cardiaca, reportes indican una prevalencia de $32 \%$ en Unidad de Cuidados Intensivos y fuera de ella. De estos pacientes, de 12 a $30 \%$ con hiperglucemia intray postoperatoria no se sabían diabéticos antes de la cirugía, este estado es por estrés, $60 \%$ de los pacientes admitidos con nueva hiperglucemia se confirman diabéticos en el lapso de un año. La hemoglobina glucosilada permite diferenciar la hiperglucemia por estrés de la diabetes no diagnosticada.

Por otra parte, hasta $25 \%$ de los pacientes con diabetes tipo 2 pueden requerir cirugía en algún momento de su vida relacionada a complicaciones crónicas que afectan los sistemas cardiovascular, oftálmico, renal y ortopédico. El riesgo de complicaciones postoperatorias (gastroparesia, cardiovasculares, infección) en pacientes con diabetes tipo 2 es mayor que en los pacientes no diabéticos reportados en estudios a gran escala. ${ }^{2,10,12}$ Karamanos y colaboradores demostraron mayor mortalidad y complicaciones cardiovasculares y renales después de colecistectomía en pacientes diabéticos, posiblemente por diabetes avanzada o comorbilidades severas, ${ }^{13}$ cuando persiste la hiperglucemia por estrés ( $>180 \mathrm{mg} / \mathrm{dL}$ ) hay efectos deletéreos, como trastornos inmunes, infecciones, trombosis, accidente vascular cerebral, infarto agudo al miocardio, estrés oxidativo y aumento de producción de especies de oxígeno reactivas. ${ }^{14}$

\section{Fisiopatología}

Durante el ayuno, el sujeto normal mantiene niveles de glucosa en plasma entre 60 a $100 \mathrm{mg} / \mathrm{dL}$. La hiperglucemia en el perioperatorio se puede presentar por estrés quirúrgico y anestésico debido a una alteración en la regulación del balance entre la producción de glucosa hepática y su utilización en tejidos periféricos: un aumento en la secreción de hormonas contrarreguladoras (catecolaminas, cortisol, glucagón y hormona del crecimiento) ocurre y causa una excesiva liberación de citocinas inflamatorias, incluyendo el factor de necrosis tumoral alfa, interleucina 6 e interleucina $1 \mathrm{~B} \cdot{ }^{15,16} \mathrm{El}$ cortisol incrementa la producción de la glucosa hepática estimulando el catabolismo proteico y promoviendo la gluconeogénesis. ${ }^{17}$ La cirugía, su tipo y duración incrementan la secreción de glucagón, catecolaminas e inhibe la secreción de insulina por las células beta pancreáticas. ${ }^{4}$ Adicionalmente, el incremento de hormonas por el estrés aumenta la lipólisis y la concentración de ácidos grasos libres. Hay evidencia que sugiere que el factor de necrosis tumoral alfa interfiere con la síntesis y/o translocación del receptor 4 transportador 
de glucosa, reduciendo la captación de glucosa en tejidos periféricos. ${ }^{18-20}$ Estos procesos resultan en un estado de alteración en la acción de la insulina sobre la glucosa, lo que genera un estado de resistencia a la insulina, el cual es más pronunciado en el primer día del postoperatorio y que puede persistir de nueve a 21 días después de la cirugía. ${ }^{21}$

La carga de carbohidratos preoperatoria cada vez es más frecuente en la práctica quirúrgica, porque puede contrarrestar el estado de resistencia a la insulina que ocurre debido al estrés e inanición. Los protocolos de recuperación acelerada después de una cirugía (ERAS) promueven la administración de bebidas ricas en carbohidratos dos horas antes de la cirugía. Esto evita un estado catabólico asociado con inanición, demostrado por un aumento en la sensibilidad a la insulina y disminución del riesgo de hiper- glucemia postoperatoria. ${ }^{22,23}$ La magnitud de la respuesta contrarreguladora se relaciona a la severidad de la cirugía, el tipo y la duración de la anestesia, líquidos intraoperatorios y apoyo nutricional han sido ligados a la elevación de la glucosa y la hiperglucemia por estrés, esto último se prolonga en cirugías que involucran tórax y abdomen cuando es comparada con procedimientos periféricos. ${ }^{23}$ Los anestésicos volátiles inhiben la secreción de insulina e incrementan la producción de glucosa hepática (Figura 1). ${ }^{24-27}$

Los síntomas de la diabetes varían dependientes del nivel de la glucosa sanguínea. Algunos de los signos y síntomas de la diabetes tipo 1 y 2 son: polidipsia, poliuria, polifagia, pérdida de peso sin causa aparente, cetonas en la orina, fatiga, irritabilidad, visión borrosa, heridas que tardan en cicatrizar e infecciones frecuentes.

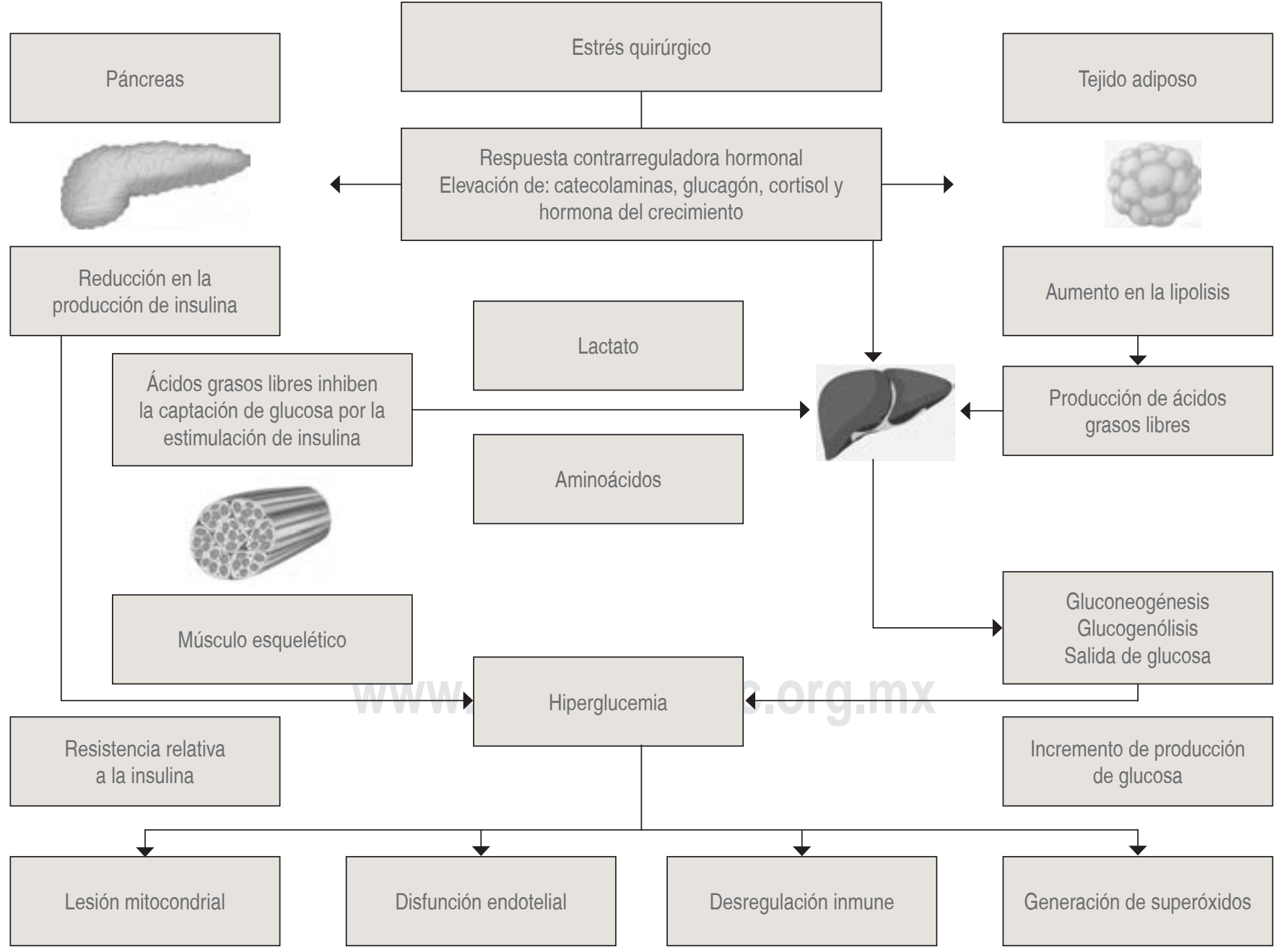

Figura 1: Hiperglucemia por estrés. ${ }^{27}$ 


\section{Clasificación}

La diabetes es una enfermedad heterogénea cuya presentación y progresión puede variar considerablemente. Se clasifica de la siguiente manera: tipo 1 y 2 , gestacional y tipos específicos debido a otras causas.

En la actualidad, se sabe que tanto los tipos 1 como 2 pueden ocurrir en cualquier grupo de edad. Los niños con diabetes tipo 1 presentan los síntomas característicos de poliuria, polidipsia y aproximadamente un tercio cetoacidosis diabética (CAD). En cambio, en los adultos puede presentarse de forma más variable, llegando a experimentar una remisión temporal de la dependencia a la insulina. De manera ocasional, los pacientes con diabetes tipo 2 pueden presentar $\mathrm{CAD}$, particularmente en minorías étnicas.

Es importante mencionar que la clasificación de la diabetes posterior al diagnóstico no siempre es sencilla. Tanto la diabetes tipo 1 como 2 poseen factores genéticos y ambientales que pueden resultar en la pérdida progresiva de masa y o función de células $\beta$, manifestándose clínicamente como hiperglucemia; una vez que ésta se produce ambos tipos de diabetes corren el riesgo de desarrollar las mismas complicaciones crónicas, aunque difiere en la tasa de progresión. ${ }^{28}$

\section{Criterios diagnósticos}

La diabetes se puede diagnosticar basándose en los criterios de glucosa plasmática, ya sea por su valor en ayunas o a las dos horas posterior a una prueba de tolerancia a la glucosa, o los criterios basados en la hemoglobina glucosilada (HbA1c). Las guías de la Asociación Americana de Diabetes (ADA, por sus siglas en inglés) aceptan los siguientes valores: glucosa plasmática en ayuno de ocho horas $\geq 7.0 \mathrm{mmol} / \mathrm{L} \mathrm{(} \geq$ $126 \mathrm{mg} / \mathrm{dL}$ ), glucosa venosa plasmática aleatoria (en un paciente con síntomas clásicos de hiperglucemia) $\geq 200 \mathrm{mg} / \mathrm{dL}$, glucosa plasmática a las dos horas de administración oral tras una prueba de tolerancia a la glucosa (carga de $75 \mathrm{~g}$ de glucosa) $\geq 11.1 \mathrm{mmol} / \mathrm{L}$ $(\geq 200 \mathrm{mg} / \mathrm{dL}), \mathrm{HbA} 1 \mathrm{c} \geq 48 \mathrm{mmol} / \mathrm{L}(\geq 6.5 \%)$. Por lo general, cualquiera de estas pruebas son igualmente apropiadas para el diagnóstico. ${ }^{28}$

Si los niveles de glucosa son altos y se encuentran en valores que no cumplan con los criterios ya mencionados, dichos resultados se clasifican como prediabetes, en caso de que los rangos de HbA1c se encuentren entre 39 a $47 \mathrm{mmol} / \mathrm{L}(5.7-6.4 \%)$, dichos pacientes poseen alto riesgo de desarrollar en el futuro diabetes mellitus tipo $2 .{ }^{29}$ Los estudios epidemiológicos que formaron la base para recomendar la HbA1c para el diagnóstico de la diabetes incluían únicamente a los adultos. Sin embargo, la ADA concluyó recientemente que puede ser empleada como diagnóstico para prediabetes o diabetes tipo 2 en niños y adolescentes. ${ }^{30}$ En condiciones asociadas al aumento en el recambio de glóbulos rojos, embarazo en el segundo y tercer trimestre, deficiencia de deshidrogenasa glucosa-6-fosfato, hemodiálisis, pérdida o transfusión de sangre reciente o terapia con eritropoyetina, sólo los criterios asociados a glucosa plasmática deben ser utilizados para el diagnóstico de diabetes. De igual manera, la HbA1c es menos confiable en estados postparto, VIH/SIDA en manejo con cierto tratamiento y anemia ferropénica. ${ }^{31}$

En el caso de que exista un diagnóstico clínico claro, éste requiere dos resultados anormales de la misma prueba o dos pruebas separadas. También se puede llegar a confirmar el diagnóstico si dos pruebas diferentes de glucosa plasmática en ayunas y HbA1c se encuentran por encima del umbral diagnóstico. ${ }^{32} \mathrm{En}$ los casos con valores plasmáticos de glucosa en ayuno por debajo de $126 \mathrm{mg} / \mathrm{dL}$ asociado a dos resultados de HbA1c mayores de $6.5 \%$ se confirma el diagnóstico de diabetes. Si los pacientes presentan resultados cerca del margen del umbral diagnóstico la prueba debe ser repetida en los siguientes tres a seis meses. En un paciente con síntomas clásicos, la medición de la glucosa plasmática en ayunas más una glucosa plasmática aleatoria $>200 \mathrm{mg} / \mathrm{dL}$ son suficientes para realizar el diagnóstico.

Las pruebas diagnósticas deben considerarse en personas con sobrepeso u obesidad, así como en aquellos adultos que tienen uno o más de los siguientes factores de riesgo: parientes en primer grado con diabetes, raza/etnia de alto riesgo, historia de enfermedad cerebral vascular, hipertensión arterial, dislipidemia, mujeres son síndrome de ovario poliquístico, sedentarismo y acantosis. Se recomienda que los pacientes con prediabetes deben someterse a las pruebas anualmente, las mujeres con diagnóstico de diabetes gestacional deben ser analizadas de por vida al menos cada tres años, el resto de la población sin factores de riesgo debe comenzar a los 45 años, de ser normales las pruebas deben repetirse con un mínimo de intervalos de tres años.

\section{Manejo de la diabetes}

El control glucémico se puede lograr por diferentes medios dependiendo la etiología de la enfermedad. Los hipoglucemiantes son comúnmente utilizados para controlar la diabetes tipo 2 junto con el cambio 
en el estilo de vida. La metformina se usa típicamente como monoterapia y primera línea en la diabetes tipo 2, por su eficacia, accesibilidad económica y pocos efectos secundarios. Dependiendo del control glucémico, puede llegar a ser necesario el empleo de terapia inyectable o combinada, incluyendo la insulina. ${ }^{33,34}$

Uno de los principales objetivos del tratamiento hipoglucemiante se basa en la reducción de las complicaciones a largo plazo. Un reciente metaanálisis realizado en el Reino Unido llevó a cabo comparaciones directas entre la monoterapia y las terapias combinadas, esta revisión sistemática continúa respaldando a la metformina como fármaco de primera línea, debido a sus beneficios sobre la HbA1c, el peso y la mortalidad cardiovascular frente al empleo de las sulfonilureas. ${ }^{35,36}$

Con respecto a la reducción del peso, se demostró que tanto las tiazolidinedionas como las sulfonilureas y la insulina llegan a disminuirlo hasta por $5 \mathrm{~kg}$. Respecto a los valores de HbA1c, los inhibidores del cotransportador sodio-glucosa tipo 2 (SGLT-2) llegan a reducir sus valores de manera similar a la metformina, no así los inhibidores de la dipeptidil peptidasa-4 (DPP-4) la reducen en menor proporción que la metformina y sulfonilureas. Respecto a la presión arterial sistólica, los inhibidores de la SGLT-2 y los agonistas del receptor de GLP-1 llegan a reducirla de 3 a $5 \mathrm{mmHg}$, sin llegar a presentar efectos concomitantes sobre la frecuencia cardiaca. ${ }^{37,38}$ La combinación de metformina más un agonista del receptor de GLP-1 se asoció con menor riesgo de hipoglucemia que la metformina más insulina premezclada o basal, favoreciendo sus efectos sobre el peso. ${ }^{39,40}$

Los diferentes mecanismos de acción sobre cada una de estas terapias producen diversos efectos, perfiles de tolerancia y beneficios adicionales más allá del control glucémico. Los resultados demostrados en la revisión sistémica realizada por Shubing Jia y colaboradores ${ }^{41}$ mostraron que la terapia combinada con miméticos de incretinas y SGLT-2 fue superior respecto a la monoterapia en términos de HbA1c. La combinación de insulina y metformina se asocia con mejor control glucémico, disminuye la dosis diaria de insulina, menor riesgo de hipoglucemia y aumento de peso. La combinación de los inhibidores de la DPP-4 e insulina parecen presentar beneficios sobre el control glucémico sin presentar efectos adversos. Nuevos medicamentos como los agonistas del receptor de GLP-1, los inhibidores del SGLT-2 poseen perfiles con beneficios aceptables sobre la reducción de peso cuando se agregan a la terapia con insulina. De acuerdo con los resultados obtenidos en dicho metaa- nálisis, se encontró que los miméticos de incretinas combinados con metformina mejoran los niveles de HbA1c, siendo superior su efecto que en combinaciones de metformina con inhibidores de SGLT-2 y DPP-4. En resumen, tanto los inhibidores de SGLT-2 y los agonistas del receptor GLP-1 poseen ventajas en términos de eficacia y seguridad. ${ }^{16,17,41-43}$

Ahora que conocemos la fisiopatología, los criterios diagnósticos y el mecanismo de acción de los fármacos con base en el tratamiento de la diabetes, hablaremos del manejo perioperatorio del paciente diabético. La importancia de un control adecuado de los niveles de glucosa en el perioperatorio radica en que el trauma quirúrgico y la anestesia alteran la regulación hormonal de la glucosa y los niveles elevados de ésta conducen a disfunción endotelial, inmunológica y daño celular. Los pacientes diabéticos sometidos a cirugía tienen una tasa de morbimortalidad más alta y un aumento en el número de días de estancia intrahospitalaria al compararlos con los pacientes no diabéticos sometidos al mismo procedimiento quirúrgico.

\section{Preoperatorio}

La valoración inicial de nuestros pacientes comienza desde el momento en que observamos por primera vez al paciente, y aunque los escenarios clínicos pueden ser infinitos, en la mente del anestesiólogo reina la primicia de identificar todos los posibles riesgos transanestésicos y postoperatorios. El conocimiento de los antecedentes clínicos del paciente nos dará las herramientas para un adecuado e individualizado manejo transanestésico. Cuando se trate de cirugía electiva es importante conocer al paciente diabético previo a la intervención quirúrgica con el objetivo de optimizar la condición base, identificar riesgos e informarle del manejo que se llevará en el perioperatorio. Es muy importante asegurarnos que la enfermedad se encuentra controlada. Para la cirugía electiva se recomienda que la $\mathrm{HbA} 1 \mathrm{c}$ sea $<8.5 \%$, de lo contrario se debe diferir hasta mejorar el control. ${ }^{44}$

Los puntos específicos por identificar en la valoración preanestésica son: ${ }^{45}$

1. Tiempo de evolución de la enfermedad.

2. Existencia de daño a órgano blanco (valoración de riesgo cardiovascular, tasa de filtración glomerular, enfermedad vascular periférica, neuropatía, retinopatía).

3. Medicación actual y pasada. Apego al tratamiento.

4. Índice de masa corporal. 
Tabla 1: Ajuste farmacológico en el perioperatorio. ${ }^{46}$

\begin{tabular}{|c|c|c|c|}
\hline Fármaco & Día previo a la cirugía & \multicolumn{2}{|c|}{ Día de la cirugía } \\
\hline Horario de la cirugía & & Matutina & Vespertina \\
\hline $\begin{array}{l}\text { Sulfonilureas } \\
\text { Metilglinidas }\end{array}$ & $\begin{array}{l}\text { Toma habitual } \\
\text { Toma habitual }\end{array}$ & $\begin{array}{l}\text { Omitir dosis matutina } \\
\text { Omitir dosis matutina }\end{array}$ & $\begin{array}{l}\text { Omitir dosis matutina y vespertina } \\
\text { Toma matutina, sólo si desayuna }\end{array}$ \\
\hline \multicolumn{4}{|c|}{ Omitir en ayuno por riesgo de cetoacidosis } \\
\hline Inhibidores de SGLT-2 & Toma habitual & Omitir & Omitir \\
\hline \multicolumn{4}{|c|}{ Pueden continuar aún en ayuno } \\
\hline $\begin{array}{l}\text { Biguanidas } \\
\text { Inhibidores de alfa glucosidasa } \\
\text { Tiazolidinedionas } \\
\text { Análogos de GLP-1 } \\
\text { Inhibidores de DPP-4 }\end{array}$ & $\begin{array}{l}\text { Toma habitual } \\
\text { Toma habitual } \\
\text { Toma habitual } \\
\text { Toma habitual } \\
\text { Toma habitual }\end{array}$ & $\begin{array}{c}\text { Omitir o tomar } \\
\text { Omitir dosis matutina } \\
\text { Toma habitual } \\
\text { Toma habitual } \\
\text { Toma habitual }\end{array}$ & $\begin{array}{l}\text { Omitir o tomar } \\
\text { Toma habitual } \\
\text { Toma habitual } \\
\text { Toma habitual } \\
\text { Toma habitual }\end{array}$ \\
\hline
\end{tabular}

5. Valor de hemoglobina glucosilada.

6. Resultado de monitoreo de glucemia en casa.

Laboratorios y estudios de imagen a solicitar:

1. Electrocardiograma de 12 derivaciones.

2. Tele de tórax.

3. Glucemia en ayunas.

4. Hemoglobina glucosilada.

5. Creatinina sérica.

6. Electrólitos séricos.

7. Examen general de orina.

\section{Programación quirúrgica}

Idealmente los pacientes diabéticos deben ser intervenidos a primera hora de la mañana, con el objetivo de disminuir el periodo de ayuno y minimizar los cambios en su rutina habitual. Es recomendable sugerir a los pacientes portar tabletas de glucosa para ingerirlas en caso de que sientan síntomas de hipoglucemia en las horas previas a la cirugía. ${ }^{27}$

\section{Ajuste farmacológico en el perioperatorio}

El objetivo es evitar los periodos de hipo- e hiperglucemia en la fase de ayuno hasta que el paciente pueda ingerir líquidos y alimentos normalmente (Tablas 1 y 2). ${ }^{46}$

\section{Manejo transanestésico}

El objetivo del manejo intraoperatorio es mantener niveles adecuados de glucosa, concentraciones de electrólitos normales, garantizar función cardiovascular óptima y adecuada perfusión renal. Son recomendables niveles de glucosa entre $100-180 \mathrm{mg} / \mathrm{dL}$, y de hasta $220 \mathrm{mg} / \mathrm{dL}$ en casos en los que el paciente tiene previamente un control deficiente de la enfermedad. Valores de 140-180 mg/dL se recomiendan en el paciente crítico. El monitoreo de la glucosa capilar debe realizarse previo a la inducción anestésica y posteriormente cada 1-2 horas durante la cirugía. ${ }^{27,44,45}$

\section{Manejo de hiperglucemia transoperatoria}

Si la glucemia excede de $220 \mathrm{mg} / \mathrm{dL}$, de ser posible se recomienda medir niveles de cetona en sangre $(>3$ $\mathrm{mmol} / \mathrm{L})$ o en orina $(>2 \times)$ para descartar cetoacidosis, cuya tríada es cetonemia $>3 \mathrm{mmol} / \mathrm{L}$, glucemia $>200 \mathrm{mg} / \mathrm{dL}$, bicarbonato $<15 \mathrm{mmol} / \mathrm{L}$ o $\mathrm{pH}<7.3$.

En pacientes con diabetes tipo 1 e hiperglucemia $>220 \mathrm{mg} / \mathrm{dL}$, se debe administrar insulina de acción rápida vía subcutánea sin exceder 6 UI por dosis, asumiendo que cada unidad de insulina disminuirá alrededor de $50 \mathrm{mg} / \mathrm{dL}$ de glucemia. La glucosa capilar debe ser medida a la hora y sólo se debe considerar una segunda dosis pasadas dos horas de la primera administración. 
En pacientes con diabetes tipo 2 e hiperglucemia $>220 \mathrm{mg} / \mathrm{dL}$ se debe administrar insulina de acción rápida vía subcutánea a una dosis de $0.1 \mathrm{UI} / \mathrm{kg}$ de peso, sin exceder 6 UI. La glucosa capilar debe ser medida a la hora y sólo se debe considerar una segunda dosis pasadas dos horas de la primera administración. Se puede considerar el uso de una bomba de infusión en caso de persistir la hiperglucemia.

\section{Tratamiento de hipoglucemia transoperatoria}

En caso de niveles de glucosa inferiores a $70 \mathrm{mg} / \mathrm{dL}$ se recomienda administrar $100 \mathrm{~mL}$ de glucosa al $20 \%$ (20 g) vía intravenosa, en caso de niveles entre 70$90 \mathrm{mg} / \mathrm{dL}$ aplicar $50 \mathrm{~mL}$ de glucosa al 20\% (10 g) vía intravenosa.

\section{Técnica anestésica}

La técnica a elegir debe ser optimizada con el fin de que el paciente regrese a la normalidad lo más pronto posible, el consumo habitual de alimentos y la medicación de rutina. La anestesia regional sola o como parte de la analgesia multimodal tiene resultados favorables en pacientes diabéticos al disminuir el uso de opioides y sus eventos adversos, disminuye el riesgo de trombosis, la respuesta quirúrgica al estrés, así como el sangrado total y facilita el retorno temprano a la vía oral; sin embargo, el bloqueo neuroaxial se ha asociado a mayor riesgo de inestabilidad hemodinámica y absceso epidural en pacientes con neuropatía autonómica previa, por lo que su uso y riesgo-beneficio debe evaluarse individualmente en este grupo de pacientes. ${ }^{47,48}$

Se ha descrito que la dexametasona puede causar hiperglucemia, en caso de usarse se debe medir la glucosa capilar cada hora en las cuatro horas posteriores a su administración. ${ }^{49}$

\section{Manejo de líquidos}

Existe poca evidencia acerca del manejo óptimo de líquidos para pacientes diabéticos, la solución Hartman se conoce segura en pacientes diabéticos y no contribuye a hiperglucemia significativa. ${ }^{50}$

Cuando se esté usando una bomba de infusión continua de insulina, se recomienda la administración de glucosa al $5 \%$ en una solución salina al $0.45 \%$ mezclada con potasio al $0.15-0.3 \%$ (20 $\mathrm{mmol} / \mathrm{L})$, con el fin de aportar sustrato a la glucosa y disminuir proteólisis, lipolisis, cetogénesis y mantener los niveles de electrólitos dentro de los valores normales. ${ }^{7} \mathrm{El}$ volumen recomendado es de $25-50 \mathrm{~mL} / \mathrm{kg} / \mathrm{día}$, aproximadamente $83 \mathrm{~mL} / \mathrm{h}$ en un paciente de $70 \mathrm{~kg} .{ }^{51}$

\section{Manejo postoperatorio inmediato}

Al ingreso del paciente en la Unidad de Cuidados Postoperatorios se debe medir glucosa capilar nuevamente. En caso de niveles de glucosa mayores a 180 $\mathrm{mg} / \mathrm{dL}$ se recomienda administrar insulina de acción rápida vía subcutánea (Tabla 3). ${ }^{52}$

La ingesta de alimentos vía oral debe reiniciarse tan pronto como sea posible.

Metas de glucosa preprandial: $100-140 \mathrm{mg} / \mathrm{dL} \mathrm{y}$ de glucosa durante el día no mayor a $180 \mathrm{mg} / \mathrm{dL} .{ }^{52}$

\section{Complicaciones agudas}

\section{Cetoacidosis diabética}

Es un estado catabólico desencadenado por dosis inadecuada de insulina y enfermedades simultáneas como infecciones. Tiene una mortalidad significativa de 1 a $5 \%$ si no se reconoce y trata precozmente. La CAD está provocada por un déficit absoluto de insuli-

Tabla 2: Ajuste farmacológico de insulina en el perioperatorio. ${ }^{46}$

\begin{tabular}{|c|c|c|c|c|}
\hline Insulinas de acción intermedia & Sin cambios & $\begin{array}{l}50 \% \text { de la dosis matutina, sólo } \\
\text { si glucemia es > } 120 \mathrm{mg} / \mathrm{dL}\end{array}$ & $\begin{array}{l}50 \% \text { de dosis matutina, sólo si } \\
\text { glucemia es }>120 \mathrm{mg} / \mathrm{dL}\end{array}$ & $\begin{array}{l}\text { Suspender hasta } \\
\text { terminar ayuno }\end{array}$ \\
\hline Insulinas premezcladas & Sin cambios & $\begin{array}{l}50 \% \text { de la dosis, sólo si } \\
\text { glucemia es > } 120 \mathrm{mg} / \mathrm{dL}\end{array}$ & $\begin{array}{l}50 \% \text { de la dosis, sólo si } \\
\text { glucemia es > } 120 \mathrm{mg} / \mathrm{dL}\end{array}$ & \\
\hline
\end{tabular}


Tabla 3: Algoritmo de insulina subcutánea. ${ }^{52}$

\begin{tabular}{cccc}
\hline $\begin{array}{c}\text { Glucemia } \\
\text { (mg/dL) }\end{array}$ & $\begin{array}{c}\text { Clase I } \\
<80 \mathrm{~kg} \\
(\mathrm{UI})\end{array}$ & $\begin{array}{c}\text { Clase II } \\
81-99 \mathrm{~kg} \\
(\mathrm{UI})\end{array}$ & $\begin{array}{c}\text { Clase III } \\
>100 \mathrm{~kg} \\
(\mathrm{UI})\end{array}$ \\
\hline$<180$ & 0 & 0 & 0 \\
$181-200$ & 1 & 2 & 4 \\
$201-250$ & 2 & 4 & 6 \\
$>250$ & $\begin{array}{c}\text { Iniciar } \\
\text { infusión }\end{array}$ & $\begin{array}{c}\text { Iniciar } \\
\text { infusión }\end{array}$ & $\begin{array}{c}\text { Iniciar } \\
\text { infusión }\end{array}$ \\
\hline
\end{tabular}

na, con un incremento concomitante de las hormonas contrainsulares (glucagón, adrenalina, cortisol y hormona del crecimiento). La hiperglucemia secundaria provoca diuresis osmótica que genera una abundante pérdida de agua y electrólitos por la orina. Se genera un estado lipolítico, con aumento de los ácidos grasos libres que son transportados hasta el hígado, donde se metabolizan dando lugar a los cuerpos cetónicos. La acumulación de estos últimos ocasiona una acidosis metabólica orgánica grave. La tríada característica es: hiperglucemia, acidosis metabólica de anión gap elevado y cetosis. El cuadro clínico incluye síntomas de hiperglucemia, dolor abdominal, náuseas, vómitos, deshidratación que puede ser severa hasta insuficiencia renal, taquipnea, respiración de Kussmaul, hipotermia y nivel de conciencia alterado. En el tratamiento es clave la administración intravenosa de insulina y la fluidoterapia intravenosa. ${ }^{53}$

\section{Hipoglucemia}

Caracterizada por la tríada de Whipple: síntomas de hipoglucemia, glucemia plasmática $<70 \mathrm{mg} / \mathrm{dL}$ y alivio de los síntomas con la administración de glucosa.

El tratamiento es la administración de glucosa, si el paciente está despierto y puede ingerir, se administran 15 g de glucosa; bajo anestesia o en inconciencia se dan vía intravenosa $25 \mathrm{~g}$ y se realiza un control de glucemia capilar cada 5 a 10 minutos hasta la resolución. ${ }^{54}$

\section{CONCLUSIONES}

Hasta $25 \%$ de los pacientes quirúrgicos pueden ser diabéticos, en México es una de las primeras causas de morbimortalidad en la población en general con incidencia y prevalencia en aumento. Es responsabilidad del equipo quirúrgico contar con los recursos necesarios para diagnosticar y tratar a tiempo los eventos de hiperglucemia en el perioperatorio, con el fin de disminuir las complicaciones relacionadas.

\section{REFERENCIAS}

1. Umpierrez GE, Isaacs SD, Bazargan N, You X, Thaler LM, Kitabchi AE. Hyperglycemia: an independent marker of inhospital mortality in patients with undiagnosed diabetes. J Clin Endocrinol Metab. 2002; 87 (3): 978-982.

2. Frisch A, Chandra P, Smiley D, Peng L, Rizzo M, Gatcliffe C et al. Prevalence and clinical outcome of hyperglycemia in the perioperative period in noncardiac surgery. Diabetes Care. 2010; 33 (8): 1783-1788.

3. Kotagal M, Symons RG, Hirsch IB, Umpierrez GE, Dellinger EP, Farrokhi ET et al. Perioperative hyperglycemia and risk of adverse events among patients with and without diabetes. Ann Surg. 2015; 261 (1): 97-103.

4. Farrokhi F, Smiley D, Umpierrez GE. Glycemic control in nondiabetic critically ill patients. Best Pract Res Clin Endocrinol Metab. 2011; 25 (5): 813-824.

5. Furnary AP, Gao G, Grunkemeier GL, Wu Y, Zerr KJ, Bookin $\mathrm{SO}$ et al. Continuous insulin infusion reduces mortality in patients with diabetes undergoing coronary artery bypass grafting. J Thorac Cardiovasc Surg. 2003; 125 (5): 1007-1021.

6. Umpierrez GE, Smiley D, Jacobs S, Peng L, Temponi A, Mulligan $\mathrm{P}$ et al. Randomized study of basal-bolus insulin therapy in the inpatient management of patients with type 2 diabetes undergoing general surgery (RABBIT 2 surgery). Diabetes Care. 2011; 34 (2): 256-261.

7. Cook G, Gall I. Anaesthetic Management of the patients with diabetes. Anaesth Intensive Care Med. 2011; 12: 438.

8. Dimas AS, Lagou V, Barker A, Knowles JW, Magi R, Hivert MF et al. Impact of type 2 diabetes susceptibility variants on quantitative glycemic traits reveals mechanistic heterogeneity. Diabetes. 2014; 63 (6): 2158-2171.

9. Saeedi P, Petersohn I, Salpea P, Malanda B, Karuranga S, Unwin $\mathrm{N}$ et al. Global and regional diabetes prevalence estimates for 2019 and projections for 2030 and 2045: Results from the International Diabetes Federation Diabetes Atlas, 9th edition. Diabetes Res Clin Pract. 2019; 157: 107843.

10. Clement S, Braithwaite SS, Magee MF, Ahmann A, Smith EP, Schafer RG et al. Management of diabetes and hyperglycemia in hospitals. Diabetes Care. 2004; 27 (2): 553-591.

11. Stacey RB, Vera T, Morgan TM, Jordan JH, Whitlock MC, Hall $\mathrm{ME}$ et al. Asymptomatic myocardial ischemia forecasts adverse events in cardiovascular magnetic resonance dobutamine stress testing of high-risk middle-aged and elderly individuals. J Cardiovasc Magn Reson. 2018; 20 (1): 75.

12. Halkos ME, Lattouf OM, Puskas JD, Kilgo P, Cooper WA, Morris CD et al. Elevated preoperative hemoglobin A1c level is associated with reduced long-term survival after coronary artery bypass surgery. Ann Thorac Surg. 2008; 86 (5): 14311437.

13. Karamanos E, Sivrikoz E, Beale E, Chan L, Inaba K, Demetriades D. Effect of diabetes on outcomes in patients undergoing emergent cholecystectomy for acute cholecystitis. World J Surg. 2013; 37 (10): 2257-2264.

14. Mongkolpun W, Provenzano B, Preiser JC. Updates in glycemic management in the hospital. Curr Diab Rep. 2019; 19 (11): 133.

15. American Diabetes Association. Diagnosis and classification of diabetes mellitus. Diabetes Care. 2010; 33 (Suppl 1): S62-S69.

16. Esposito K, Nappo F, Marfella R, Giugliano G, Giugliano $\mathrm{F}$, Ciotola $\mathrm{M}$ et al. Inflammatory cytokine concentrations 
are acutely increased by hyperglycemia in humans: role of oxidative stress. Circulation. 2002; 106 (16): 2067-2072.

17. Chan TM. The permissive effects of glucocorticoid on hepatic gluconeogenesis. Glucagon stimulation of glucose-suppressed gluconeogenesis and inhibition of 6-phosphofructo-1-kinase in hepatocytes from fasted rats. J Biol Chem. 1984; 259 (12): 7426-7432.

18. Roden M, Price TB, Perseghin G, Petersen KF, Rothman DL, Cline GW et al. Mechanism of free fatty acid-induced insulin resistance in humans. J Clin Invest. 1996; 97 (12): 2859-2865.

19. Dresner A, Laurent D, Marcucci M, Griffin ME, Dufour S, Cline GW et al. Effects of free fatty acids on glucose transport and IRS-1-associated phosphatidylinositol 3-kinase activity. J Clin Invest. 1999; 103 (2): 253-259.

20. Hotamisligil GS, Murray DL, Choy LN, Spiegelman BM. Tumor necrosis factor alpha inhibits signaling from the insulin receptor. Proc Natl Acad Sci U S A. 1994; 91 (11): 4854-4858.

21. Thorell A, Efendic S, Gutniak M, Haggmark T, Ljungqvist O. Insulin resistance after abdominal surgery. Br J Surg. 1994; 81 (1): 59-63.

22. Svanfeldt M, Thorell A, Hausel J, Soop M, Nygren J, Ljungqvist O. Effect of "preoperative" oral carbohydrate treatment on insulin action--a randomised cross-over unblinded study in healthy subjects. Clin Nutr. 2005; 24 (5): 815-821

23. Awad S, Varadhan KK, Ljungqvist O, Lobo DN. A metaanalysis of randomised controlled trials on preoperative oral carbohydrate treatment in elective surgery. Clin Nutr. 2013; 32 (1): 34-44.

24. Clarke RS. The hyperglycaemic response to different types of surgery and anaesthesia. Br J Anaesth. 1970; 42 (1): 45-53.

25. Rehman HU, Mohammed K. Perioperative management of diabetic patients. Curr Surg. 2003; 60 (6): 607-611.

26. Desborough JP, Jones PM, Persaud SJ, Landon MJ, Howell SL. Isoflurane inhibits insulin secretion from isolated rat pancreatic islets of Langerhans. Br J Anaesth. 1993; 71 (6): 873-876.

27. Duggan EW, Carlson K, Umpierrez GE. Perioperative hyperglycemia management: an update. Anesthesiology. 2017; 126 (3): 547-560. doi: 10.1097/ALN.0000000000001515.

28. American Diabetes Association. 2. Classification and diagnosis of diabetes: standards of medical care in diabetes-2020. Diabetes Care. 2020; 43 (Suppl 1): S14-S31.

29. Robinson C, McGinlay M, Mruthunjaya S. Perioperative management of diabetes. Anaesth Intensive Care Med. 2020. doi: 10.1016/j.mpaic.2020.08.001.

30. Arslanian S, Bacha F, Grey M, Marcus MD, White NH, Zeitler P. Evaluation and management of youth-onset type 2 diabetes: a position statement by the american diabetes association. Diabetes Care. 2018; 41 (12): 2648-2668.

31. Welsh KJ, Kirkman MS, Sacks DB. Role of glycated proteins in the diagnosis and management of diabetes: research gaps and future directions. Diabetes Care. 2016; 39 (8): 1299-1306.

32. Selvin E, Wang D, Matsushita K, Grams ME, Coresh J. Prognostic implications of single-sample confirmatory testing for undiagnosed diabetes: a prospective cohort study. Ann Intern Med. 2018; 169 (3): 156-164.

33. Selvin E. Are there clinical implications of racial differences in HbA1c? A difference, to be a difference, must make a difference. Diabetes Care. 2016; 39 (8): 1462-1467.

34. Lean ME, Leslie WS, Barnes AC, Brosnahan N, Thom G, McCombie L et al. Primary care-led weight management for remission of type 2 diabetes (DiRECT): an open-label, clusterrandomised trial. Lancet. 2018; 391 (10120): 541-551.
35. Inzucchi SE, Lipska KJ, Mayo H, Bailey CJ, McGuire DK. Metformin in patients with type 2 diabetes and kidney disease: a systematic review. JAMA. 2014; 312 (24): 2668-2675.

36. Holman RR, Coleman RL, Chan JCN, Chiasson JL, Feng $\mathrm{H}, \mathrm{Ge} \mathrm{J}$ et al. Effects of acarbose on cardiovascular and diabetes outcomes in patients with coronary heart disease and impaired glucose tolerance (ACE): a randomised, doubleblind, placebo-controlled trial. Lancet Diabetes Endocrinol. 2017; 5 (11): 877-886.

37. Kernan WN, Viscoli CM, Furie KL, Young LH, Inzucchi SE, Gorman M et al. Pioglitazone after ischemic stroke or transient ischemic attack. N Engl J Med. 2016; 374 (14): 1321-1331.

38. Vaccaro O, Masulli M, Nicolucci A, Bonora E, Del Prato S, Maggioni AP et al. Effects on the incidence of cardiovascular events of the addition of pioglitazone versus sulfonylureas in patients with type 2 diabetes inadequately controlled with metformin (TOSCA.IT): a randomised, multicentre trial. Lancet Diabetes Endocrinol. 2017; 5 (11): 887-897.

39. Health and Social Care Information Centre. National Diabetes Inpatient Audit (NaDIA), Open data - 2013. 2014. Available in: http://www.hscic.gov.uk/catalogue/PUB14358

40. Maruthur NM, Tseng E, Hutfless S, Wilson LM, SuarezCuervo C, Berger Z et al. Diabetes medications as monotherapy or metformin-based combination therapy for type 2 diabetes: a systematic review and meta-analysis. Ann Intern Med. 2016; 164 (11): 740-751.

41. Jia S, Wang Z, Han R, Zhang Z, Li Y, Qin X et al. Incretin mimetics and sodium-glucose co-transporter 2 inhibitors as monotherapy or add-on to metformin for treatment of type 2 diabetes: a systematic review and network meta-analysis. Acta Diabetol. 2021; 58 (1): 5-18.

42. Li T, Providencia R, Mu N, Yin Y, Chen M, Wang Y et al. Association of metformin monotherapy or combined therapy with cardiovascular risks in patients with type 2 diabetes mellitus. Cardiovasc Diabetol. 2021; 20 (1): 30.

43. Abdi H, Azizi F, Amouzegar A. Insulin monotherapy versus insulin combined with other glucose-lowering agents in type 2 diabetes: a narrative review. Int J Endocrinol Metab. 2018; 16 (2): e65600.

44. Partridge H, Perkins B, Mathieu S, Nicholls A, Adeniji $\mathrm{K}$. Clinical recommendations in the management of the patient with type 1 diabetes on insulin pump therapy in the perioperative period: a primer for the anaesthetist. Br J Anaesth. 2016; 116 (1): 18-26.

45. Guidelines for managing continuous subcutaneous insulin infusion therapy in hospitalised patients. DTN-UK.

46. Membership of the Working Party, Barker P, Creasey PE, Dhatariya K, Levy N, Lipp A et al. Peri-operative management of the surgical patient with diabetes 2015: Association of Anaesthetists of Great Britain and Ireland. Anaesthesia. 2015; 70 (12): 1427-1440. doi: 10.1111/anae.13233.

47. Dhatariya K, Levy N, Kilvert A, Watson B, Cousins D, Flanagan D et al. NHS Diabetes guideline for the perioperative management of the adult patient with diabetes. Diabet Med. 2012; 29 (4): 420-433.

48. National Collaborating Centre for Acute Care (UK). Preoperative tests: the use of routine preoperative tests for elective surgery. London: National Collaborating Centre for Acute Care (UK); 2003. Available in: www.nice.org.uk/ guidance/

49. Cook TM, Counsell D, Wildsmith JA; Royal College of Anaesthetists Third National Audit Project. Major complications of central neuraxial block: report on the Third National Audit 
An Med ABC. 2021; 66 (3): 195-204

Project of the Royal College of Anaesthetists. Br J Anaesth. 2009; 102 (2): 179-190. Available in: www.rcoa.ac.uk/nap3

50. Simpson AK, Levy N, Hall GM. Peri-operative i.v. fluids in diabetic patients--don't forget the salt. Anaesthesia. 2008; 63 (10): 1043-1045.

51. National Institute for Health and Care Excellence. Intravenous fluid therapy in adults in hospital. NICE guideline CG174, December 2013. Available in: www.nice.org. uk/guidance/cg174/
52. Joslin Diabetes Center and Joslin Clinic guideline for inpatient management of surgical and ICU patients with diabetes (pre, peri and postoperative care) 1230 2015; updated 04/22/19.

53. Kitabchi AE, Umpierrez GE, Miles JM, Fisher JN. Hyperglycemic crises in adult patients with diabetes. Diabetes Care. 2009; 32 (7): 1335-1343.

54. Kittah NE, Vella A. Management of endocrine disease: pathogenesis and management of hypoglycemia. Eur J Endocrinol. 2017; 177 (1): R37-R47. 